\title{
PHYSICAL ACTIVITY LEVELS AND ACTIVITY PREFERENCES OF A COHORT OF SOUTH AFRICAN INDIVIDUALS LIVING WITH HIV
}

\begin{abstract}
Physical inactivity is a risk factor for many lifestyle diseases. It is still poorly understood in individuals living with HIV. The aims of this study were to evaluate the physical activity levels of individuals living with HIV on antiretroviral therapy (ARV) and to assess their physical activity preferences. An observation study was done from October 2010 to June 2012 at a large urban HIV clinic in Johannesburg where 205 individuals were consecutively sampled. Physical activity was assessed with the Yamax SW200 pedometer over a seven day period. The mean age of the sample was 38 ( \pm 9.5$)$ years, $C D_{4}$ count 285.1 ( \pm 157.9$)$ and time on ARV $8.7( \pm 2.3)$ months. Physical activity of the sample was reduced at 7673.2 ( \pm 4017.7) steps per day with women

Roos R, MSc Physiotherapy'; Myezwa $\mathrm{H}, \mathrm{PhD}^{1}$; Van Aswegen $\mathrm{H} \mathrm{PhD}^{1}$

1 Physiotherapy Department, Faculty of Health Sciences, University of the Witwatersrand. walking less than men [6993.3 ( \pm 3462.6$)$ and $10076.3( \pm 4885.6)$ respectively]. Eight individuals (3.9\%) did formal sporting activities and a hundred and twenty three individuals (60\%) did some form of exercise. Walking $(45.5 \%, n=56)$ and running $(26.8 \%, n=33)$ were most frequently reported. Physical inactivity was present in the majority of the sample and interventions including activity preferences of individuals could be strategies to address this health concern.
\end{abstract}

KEY WORDS: PHYSICAL ACTIVITY, PEDOMETER, ACTIVITY PREFERENCES, HIV.

\section{INTRODUCTION}

Physical inactivity is a health concern worldwide due to the increased burden related to this risk factor for many chronic non-communicable diseases (Lee et al 2012). The World Health Survey reported the crude prevalence rates for physical inactivity in South Africa to be $43 \%$ for men and $47 \%$ for women in $2002-2003$ (Guthold et al 2008). If the burden of physical inactivity is reviewed in relation to noncommunicable diseases and ischaemic heart disease (IHD) is used as an example, $30 \%$ of IHD cases in South Africa are attributed to physical inactivity

\section{Correspondence Author:}

R Roos

Department of Physiotherapy,

Faculty of Health Sciences,

University of the Witwatersrand,

Johannesburg, South Africa

Email: ronel.roos@wits.ac.za
(Joubert et al 2007) compared to international statistics of $6 \%$ (Lee et al 2012). Screening of physical activity levels is said to be the first step in effectively managing this health concern as it provides information on the status quo and provides means for comparing different populations (Hallal et al 2012). Objective devices such as accelerometers and/or pedometers are the preferred suggested means to gather such data (Hallal et al 2012; Tudor-Locke and Lutes 2009).

Physical activity has been well researched in the general population but is still poorly understood in the HIV population. This is of concern particularly in the South African context due to the number of individuals living with HIV. An estimated $17 \%$ of South Africans are living with HIV according to the 2011 World prevalence rates of HIV and only Swaziland, Botswana and Lesotho report higher statistics (USAID 2011). In the South African context HIV prevalence remains dispropor- tionally high in females in comparison to males and it is estimated that $1 / 5$ of childbearing aged women are HIV positive (Statistics South Africa 2010). The likelihood of South African individuals infected with HIV to live longer is also now more probable. This is due to antiretroviral initiation guidelines currently being aligned with international guidelines (Mayosi et al 2012). In this context, the current limited published literature related to physical activity creates an opportunity for investigative research into the different facets of physical activity and to provide information on the South African situation. The aim of this study was therefore twofold: firstly to determine the physical activity levels of a cohort of South African individuals living with HIV initiated on highly active antiretroviral therapy (HAART); and secondly to evaluate said individuals' physical activity preferences to provide information on modes of activity. 


\section{METHOD}

\section{STUDY DESIGN AND PARTICIPANT} DESCRIPTION

An observational study was carried out at a large urban HIV outpatient clinic in Johannesburg South Africa from October 2010 to June 2012. Participants were sampled consecutively according to inclusion and exclusion criteria. The information presented in this paper forms part of a much larger study investigating the risk factors for IHD in individuals who are HIV positive. The inclusion, exclusion criteria and sample size calculation are therefore based on the objectives of the larger study. Individuals were included if they were between 20 - 65 years of age, on HAART treatment for six to twelve months and ambulatory without an assistive device. They were excluded if they had any past medical history of cardiovascular disease, difficulty walking, were currently pregnant, presented with any acute illness and/or current active opportunistic infection or were emotionally unstable. Sample size was calculated at 195 study participants using prevalence rates for hypertension in the South African context as a guide. No prevalence rates for IHD in the general and/ or HIV population in South Africa were available at the start of the project (Steyn, 2008). Alpha was set at 5\% and power at $80 \%$. The sample was increased with a factor of 100/95 to allow for any loss to follow-up of participants accounting for a final sample size of 205 .

\section{Procedure}

The study was approved by the University of the Witwatersrand Human Ethics committee. Permission was received from the hospital, clinic management and all participants gave informed consent prior to participation. Two research assistants gained informed consent from study participants. Figure $\mathbf{1}$ is a flow diagram of the procedure related to the study.

Figure 2 is a flow diagram that summarises retention of individuals from interest to participation until attendance of the second assessment visit. Table I consists of the demographics related to the study participants.

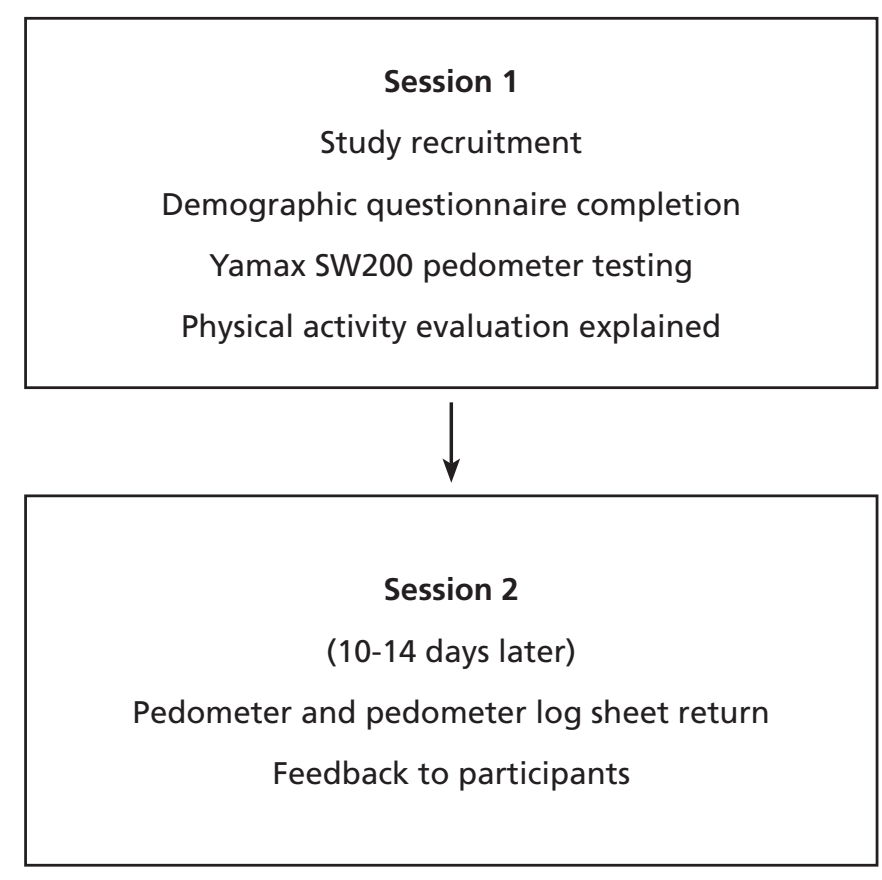

Figure 1: Flow diagram of study procedure.

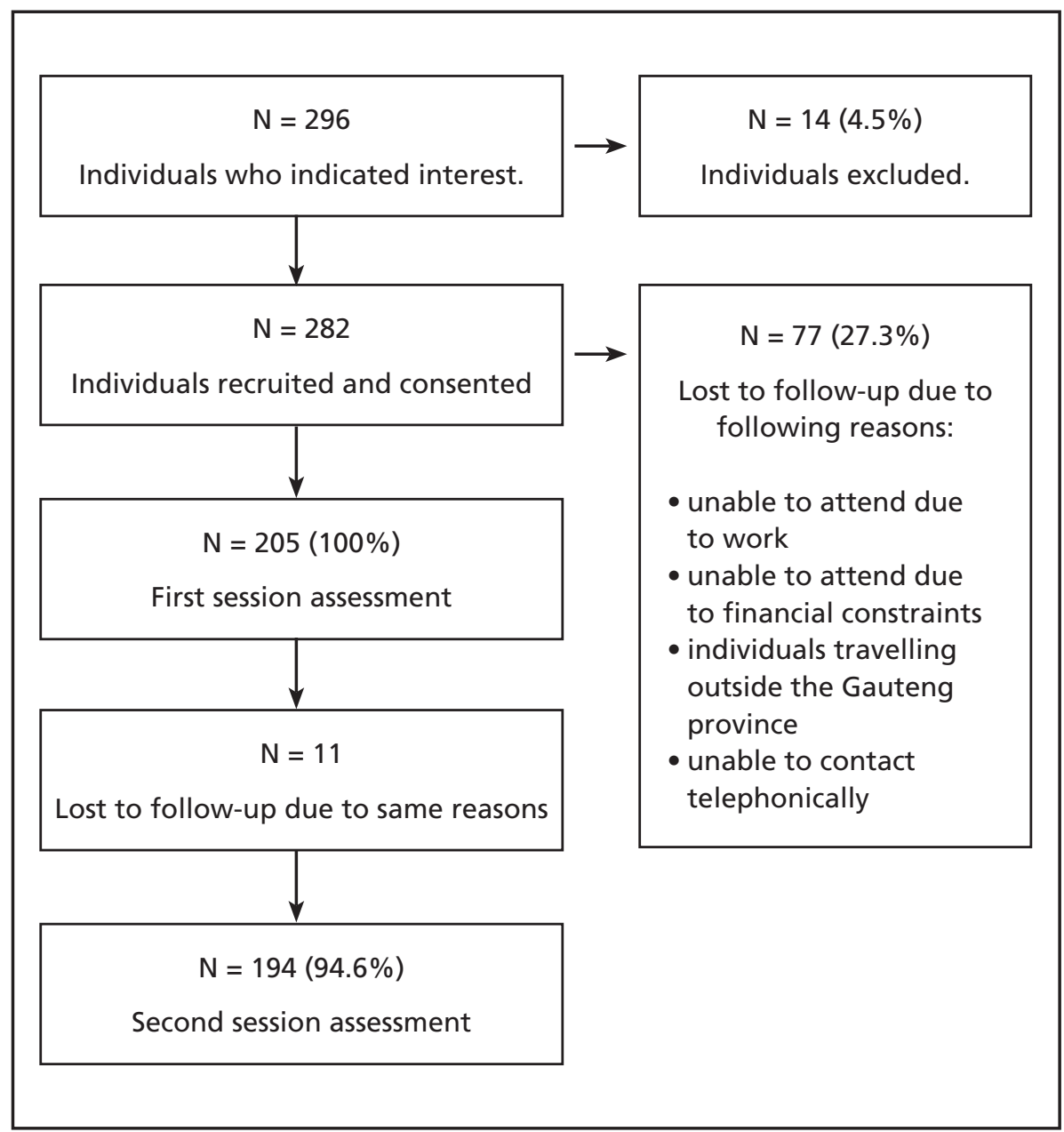

Figure 2: Flow diagram to illustrate participants' retention from interest to assessment. 
Each participant completed a demographic questionnaire that included questions pertaining to demographic background, HIV history and physical activity preferences. Study participants were asked two standardised questions to evaluate their mode of physical activity preferences: "Do you participate in any formal sport activity?" and "Do you do any exercises on your own?" Participants could answer yes or no to the questions posed and were then asked to elaborate on their answers. A "formal sport activity" was considered any physical activity that was being supervised at the time such as by a coach and/ or physical trainer. Individuals' latest CD4 count value were collected from their clinic file and/or clinic laboratory database. Time spent on HAART and details of specific HAART medication were collected from each participant and his/her clinic file.

Physical activity level was assessed using the Yamax SW200 pedometer to provide information on walking behaviour (daily step count). The Yamax SW200 pedometer is considered a valid tool for assessing ambulation physical activity during free-living physical activity (Tudor-Locke et al 2002). It is also often used as the criterion when evaluating other pedometers (TudorLocke and Lutes 2009). The convergent validity of the Yamax SW200 pedometer with a dual-mode CSA accelerometer was noted to be high at $\mathrm{r}=0.74-0.86$ (Tudor-Locke et al 2002). Participants were asked to wear the pedometer for seven consecutive days from getting up in the morning until going to bed at night and to document their daily steps on a physical activity log sheet at night when they removed the pedometer. The physical activity log sheet was available in an English and isiZulu format and participants could choose which form they wanted to complete. They were encouraged not to alter their normal physical activity routine during the evaluation period. The hip-mounted pedometer placement was standardised by instructing participants to place it on a belt or waistband, on the right side in the midline of the thigh, consistent with manufacturer's recommendations. Each morning prior to positioning the pedo- meter they had to reset the pedometer monitor to zero. Reactivity related to the physical activity assessment method was calculated following a pilot study done on 24 individuals. No significant alteration $(\mathrm{p}=0.4)$ in physical activity level was observed between the first and last day of assessment in participants when wearing the hip-mounted pedometer and documenting their findings on a physical activity log sheet during the pilot study. The accuracy of the pedometers was evaluated when participants were wearing them prior to assessing their physical activity levels during the seven day period. This was assessed by means of a 10 metre walking trial in which pedometer registered steps were compared to actual counted steps. The walking trial was done twice and the best agreement evaluated in the pilot study. The association between the pedometer counted steps and actual counted steps was moderate $(r=0.6$ with $p=0.03)$. The participants attended two sessions with the first author and all consultations occurred in a private consultation room within the clinic. A research assistant acted as translator during the study if a participant was unable to understand English or Afrikaans.

\section{STATISTICAL ANALYSIS}

Data analysis was done with STATA 12.0 and IBM SPSS 20. Data were evaluated for normal distribution. Continuous data e.g. pedometer step count were summarised as means and standard deviations. Categorical data e.g. gender, physical activity categories and exercise preference responses were summarised as frequencies and percentages. A review of participants' responses provided the information regarding type of sporting activities and exercises performed by individuals. The following pedometer physical activity categories were used as a guide during interpretation of results: "sedentary" less than 5000 steps per day, "light active" between 5000 7499 steps per day, "somewhat active" between 7500-9999 steps per day, "active" between 10000-12499 steps per day and "very active" more than 12500 steps per day (Tudor-Locke and Bassett 2004). Findings were reported to one decimal value.
The significance of the study was set at $\mathrm{p}<0.05$.

\section{RESULTS}

The sample consisted mostly of females $[77.1 \%(n=158)]$. The majority of participants had a secondary school education $[46.3 \%(\mathrm{n}=95)]$, had dependents $[85.4 \%(n=158)]$ and were employed $[56.1 \%(n=115)]$. The majority of par ticipants were on the current first line ARV regimen of South Africa that consists of Lamivudine, Efavirenz, and Tenofovir [67.8\% $(n=139)]$. The majority of participants did not participate in a formal sporting activity [96.1\% $(n=197)]$ but two-third of the sample tried to be active by doing exercises on their own [60\% $(n=123)]$.

Physical activity data for 195 participants were available for analysis due to the following reasons: three participants' data were excluded during analysis due to not completing seven days of pedometer assessment, seven participants did not attend their second visit or return their pedometer and pedometer log sheet and three participants send a friend/ family member to return their pedometer and physical activity log sheet if they could not attend their second session. The mean pedometer step count finding of the sample was $7673.2( \pm 4017.7)$ with women $(\mathrm{n}=152)$ walking less [6993.3 $( \pm 3462.6)]$ than men $(n=43)$ [10 076.3 (4885.6)]. The study participants' frequencies according to pedometer physical activity categories were as follows: $25.4 \%(n=52)$ were "sedentary", 27.8\% $(\mathrm{n}=57)$ were "light active", $20 \%(\mathrm{n}=41)$ were "somewhat active", $11.2 \%(\mathrm{n}=23)$ were considered "active" and 10.7\% $(n=22)$ were "very active". The majority of the sample therefore fell into the less than active category.

Eight participants (3.9\%) participated in formal sporting activities and one hundred and twenty three participants $(60 \%)$ incorporated some form of exercise into their life. Table 2 is an outline of the physical activity preferences of those individuals who participated in formal or self-initiated exercise.

\section{DISCUSSION}

The aim of this study was firstly to determine the physical activity level of 
a cohort of South African individuals living with HIV on HAART and secondly to evaluate said individuals' physical activity preferences to provide information on modes of activity. Ambulation physical activity (walking behaviour) was of interest in this study as it forms part of a much larger project related to ischaemic heart disease risk and lower levels of physical activity such as activities of daily living were not collected. This study contributes to the body of knowledge specific to ambulation physical activity and provides information of an urban South African group of individuals living with HIV when on HAART for six to twelve months. Male participants accumulated more steps per day compared to their female counterparts. This finding is similar to studies conducted in the general South African population concerning prevalence rates of physical inactivity and gender differences (Guthold et al 2008). The pedometer step count finding was less than that reported by Cook et al (2010) in South African individuals living in a rural area. The average step count in their sample was 12471 steps/day. It is well known that epidemiological transition that consists partly of urbanisation result in behavioural change (Mensah 2008). The change is then often noted in individuals' diet and physical activity levels. The different settings could therefore have influenced the physical activity levels due to possible differences in transportation, geographical location and living environment of participants in this study. The HIV status of the rural population was also not reported and this added possible difference in immune function between the two study populations could explain the different step count findings. Increased viral load and decreased CD4 count is associated with HIV disease progression. The participants' mean CD4 count was more than 200 cells $/ \mu \mathrm{L}$ but was still not optimal. It is known that an inverse relationship exists between physical activity levels and these two immune parameters in individuals (Bopp et al 2004). The sample was on HAART for 8.7 months and it may be possible that with longer HAART exposure their physical activity levels might improve over time due to

Table 1: Demographics of study participants $(n=205)$

\begin{tabular}{|c|c|}
\hline Variable & $\begin{array}{l}\text { Percentage }(n) \text { or } \\
\text { Mean }( \pm S D)\end{array}$ \\
\hline $\begin{array}{l}\text { Gender } \\
\text { - Male } \\
\text { - Female }\end{array}$ & $\begin{array}{l}22.9 \%(47) \\
77.1 \%(158)\end{array}$ \\
\hline $\begin{array}{l}\text { Educational level } \\
\text { - No education } \\
\text { - Primary school education } \\
\text { - Secondary school education } \\
\text { - Post-secondary school education }\end{array}$ & $\begin{array}{l}2.9 \%(6) \\
24.4 \%(50) \\
46.3 \%(95) \\
26.3 \%(54)\end{array}$ \\
\hline $\begin{array}{l}\text { Employment status } \\
\text { - Unemployed } \\
\text { - Employed } \\
\text { - Self-employed }\end{array}$ & $\begin{array}{l}40.5 \%(83) \\
56.1 \%(115) \\
3.4 \%(7)\end{array}$ \\
\hline $\begin{array}{l}\text { Participants who had dependents } \\
\text { - No } \\
\text { - Yes }\end{array}$ & $\begin{array}{l}14.6 \%(30) \\
85.4 \%(158)\end{array}$ \\
\hline $\begin{array}{l}\text { Participants who did formal sport activity } \\
\text { - No } \\
\text { - Yes }\end{array}$ & $\begin{array}{l}96.1 \%(197) \\
3.9 \%(8)\end{array}$ \\
\hline $\begin{array}{l}\text { Participants who exercised on their own } \\
\text { - No } \\
\text { - Yes }\end{array}$ & $\begin{array}{l}40 \%(82) \\
60 \%(123)\end{array}$ \\
\hline $\begin{array}{l}\text { ARV categories } \\
\text { - Lamivudine, Efavirenz, Tenofovir } \\
\text { - Lamivudine, Efavirenz, Stavudine } \\
\text { - Other }\end{array}$ & $\begin{array}{l}67.8 \%(139) \\
18.5 \%(38) \\
13.6 \%(28)\end{array}$ \\
\hline $\begin{array}{l}\text { Perceived health perception } \\
\text { - Poor } \\
\text { - Average } \\
\text { - Good } \\
\text { - Excellent }\end{array}$ & $\begin{array}{l}2.4 \%(5) \\
19.5 \%(40) \\
58.5 \%(120) \\
19.5 \%(40)\end{array}$ \\
\hline Time on ARVs & $8.7( \pm 2.3)$ \\
\hline CD4 count & $285.1( \pm 157)$ \\
\hline Age & $38.2( \pm 9.5)$ \\
\hline
\end{tabular}

stabilisation in immune parameters and improved wellness.

In some instances HAART might have negative effects on physical activity. Stavudine containing regimens are known to increase the risk of distal sensory polyneuropathy (DSP) in individuals living with HIV (Shurie and Deribew, 2010) and this could influence an individual's walking ability. The development of DSP in individuals on Stavudine might have been one of the factors that influenced the alteration of the National HAART initiation guidelines to now include Tenofovir contain- ing regimen as the first line HAART regimen in South Africa (Department of Health, 2013). A percentage of the study sample was on Stavudine but at the time of this study were not reporting any symptoms of DSP however the possibility of DSP affecting physical activity is possible (Ites et al 2011). The physical activity levels are very similar to an international HIV group. Ramirez-Marrero et al (2008) investigated the physical activity levels in 58 Hispanic adults living with HIV in San Juan, Porto Rico. They reported a mean of $7418( \pm 2714)$ steps per day with men accumulating slightly 
Table 2: Physical activity preferences of exercising study participants

\begin{tabular}{|l|l|}
\hline \multicolumn{1}{|c|}{ Physical activity mode } & \multicolumn{1}{|c|}{ Percentage (n) } \\
\hline Formal sporting activities & 8 \\
- Aerobic exercise & $25.0 \%(2)$ \\
- Soccer & $25.0 \%(2)$ \\
- Basketball & $12.5 \%(1)$ \\
- Netball & $12.5 \%(1)$ \\
- Running group & $12.5 \%(1)$ \\
- Horse jockeying & $12.5 \%(1)$ \\
\hline Self-initiated exercise & 123 \\
Individual exercise & \\
- Walking & $45.5 \%(56)$ \\
- Running & $26.8 \%(33)$ \\
- Strengthening exercises & $15.4 \%(19)$ \\
- Own aerobics & $0.1 \%(6)$ \\
- Stretching & $0.02 \%(3)$ \\
- Dancing & $0.02 \%(3)$ \\
- Jumping & $0.02 \%(2)$ \\
- Cycling & $0.02 \%(2)$ \\
- Yoga & $0.01 \%(1)$ \\
- Vibration machine & $0.01 \%(1)$ \\
\hline Social exercise & \\
- Netball & $0.02 \%(3)$ \\
- Soccer & $0.02 \%(2)$ \\
- Squash & $0.01 \%(1)$ \\
\hline Physical activity through home related activities & \\
- Housework & $0.01 \%(1)$ \\
\hline Physical activity through work related activities & $0.02 \%(2)$ \\
- Active at work & $0.01 \%(1)$ \\
\hline
\end{tabular}

less steps than the South African males at $7594( \pm 2817)$ and women slightly more at $7151( \pm 2589)$ steps/day.

The beneficial effects of exercise in individuals infected with HIV have been well reported. These effects include improvement in quality of life, cardiometabolic risk factors, cardiorespiratory fitness and muscle strength (O'Brien et al 2010). A challenge that investigators are often faced with when conducting clinical studies are study participants' adherence to exercise and withdrawal from interventions. O'Brien et al (2010) report the following reasons for non-adherence and withdrawal compiled from a review of exercise studies in individuals living with HIV. Reasons for withdrawal include: lack of interest, time and motivation; economic issues; issues related to study participants' family; transportation difficulties and sessions clashing with work schedules. Reasons related to exercise non-adherence included: illness; complaints that exercises were too difficult; time limitation; loss of interest and transportation difficulties (O'Brien et al 2010). Here loss to follow-up reasons was very similar to the responses noted above. One interesting finding during the course of the study however was the difficulty contacting individuals telephonically. The majority of participants used mobile phones as communication devices. The clinic where the study was undertaken also found contacting individuals problematic and explained that patients often buy new sim-cards where free airtime was included instead of buying airtime for an existing phone due to less cost involved and savings accrued when a new simcard is bought.

A possible strategy to lessen withdrawal and non-adherence to exercise programmes is to implement activity that individuals prefer doing. Published information on physical activity preferences in a HIV population could not be found and information in a general population is also scarce. Burton, Khan and Brown (2012) evaluated physical activity preferences in adults at risk of being inactive in a general Australian population. The format (how), location (where) and social setting (with whom) of the physical activity preferences were assessed. The researchers noted that more than $75 \%$ of respondents preferred activities at no or limited cost, $80 \%$ preferred activities that could be done close to home and $75 \%$ of respondents preferred activities that could be done alone. Similarly in this study, the majority of participants did exercise that was not supervised and could also be done on their own. They most often included activities such as walking or running. Walking has often been reported to be the preferred mode of activity in individuals with type two diabetes (Forbes et al 2010) and sedentary individuals (Booth et al 1997). This might be due to walking not being dependent on learning a new skill, having no cost implications, not dependent on belonging to an exercise group and can be done close to home. Even though participants reported walking and running as their preferred activities, the majority of the sample still accumulated less than 10000 steps per day. Both these activities are dependent on ambulation activity and are ideal for assessing and monitoring with a pedometer. If participants did these two activities regularly during the seven days of assessment it would have been reflected in their pedometer findings. It could therefore be argued that they reported some healthy activity behaviour but the actual amount of these two activities was not given.

The current study is of clinical significance as it demonstrated that most study participants attempted increasing their activity level by performing some type of exercise even though their immune parameters were not optimal. Knowledge that study participants gain during current clinic HAART initiation programmes on living a healthy lifestyle as a means of improving immune function is therefore influencing indi- 
vidual behaviour. Clinical programmes that advocate moderated intensity activity such as home-based walking could potentially be a form of activity that individuals living with HIV would adhere to as it is the preferred activity of choice and easy to self-monitor. Such programmes would also alleviate the burden on healthcare workers to care for individuals living with HIV by promoting self-monitoring. There is however a need for education and implementation of activity programmes for individuals living with HIV to improve their frequency of exercise and possibly the intensity level of activities in order to reap the reported health benefits of exercise. An interesting finding was that only four individuals reported housework, gardening and work related activity as a form of exercise. This finding may have been different if further probing questions were added to the two standardised physical activity preference questions asked. This study was performed in individuals that were on HAART for six to 12 months and physical activity findings might be different if the inclusion criteria related to HAART exposure were widened. Future studies could investigate physical activity levels related to length of HAART exposure and different agegroups. A comparative review with a general South African population could also be beneficial. Longitudinal studies that include variables such as quality of life and other non-communicable disease risk factors such as obesity and its relationship with physical activity could be suggested for future studies. Secondly, the focus of the study pertaining to physical activity preferences was to find out the mode (method) of activity and not format, location and social setting as assessed by other authors. Future studies could possibly look into the wider scope of physical activity preferences to include these components.

\section{CONCLUSION}

In conclusion, this study provides information on the physical activity levels and mode of physical activity preferences of individuals living with HIV attending an urban HIV clinic. Physical inactivity was present in the majority of study participants as they took less than the recommended 10000 steps per day. Intervention programmes addressing this risk factor for ischaemic heart disease at a primary care level could potentially lessen the future burden of disease related to inactivity.

\section{ACKNOWLEDGEMENT}

This study was possible due to support received from the Themba Lethu HIV clinic, Clinical HIV Research Unit, Right to Care and the Department of Medicine at Helen Joseph Hospital in Gauteng, South Africa. This study was supported by grants received from the University of the Witwatersrand (Medical Endowment and Faculty Research Committee grants), South African Society of Physiotherapy (Research Foundation grant), National Research Foundation (NRF) Thuthuka grant and Medical Research Council (MRC). "Any opinion, findings and conclusions or recommendations expressed in this material are those of the author(s) and therefore the NRF does not accept any liability in regard thereto. The views and opinions expressed are not those of the MRC but of the author (s) of the material produced or publicized".

\section{REFERENCES}

Booth ML, Bauman A, Owen N, Gore CJ 1997 Physical activity preferences, preferred sources of assistance and perceived barriers to increased activity among physically inactive Australians. Preventive Medicine 26 (1): 131-137.

Bopp CM, Phillips KD, Fulk LJ, Dudgeon WD, Sowell R, Hand GA 2004 Physical activity and immunity in HIV-infected individuals. AIDS Care 16 (3): 387-393.

Burton NW, Khan A, Brown WJ 2012 How, where and with whom? Physical activity context preferences of three adult groups at risk of inactivity. British Journal of Sports Medicine 46: 1125-1131.

Cook I, Alberts M, Brits JS, Choma SR, Mkhonto SS 2010 Descriptive epidemiology of ambulatory ambulatory activity in rural, black South Africans. Medicine \& Science in Sports \& Exercise 42 (7): 1261-1268

Department of Health 2013 The South African Antiretroviral treatment guidelines. http://www. doh.gov.za/docs/policy/2013/ART_Treatment Guidelines_Final_25March2013.pdf. Accessed on 2013-07-09.

Forbes CC, Plotnikoff RC, Courneya KS, Boule NG 2010 Physical activity preferences and type two diabetes: exploring demographic, cognitive and behavioural differences. The Diabetes Educator 36 (5): 801-815.
Guthold R, Ono T, Strong KL, Chatterji S, Morabia A 2008 Worldwide variability in physical inactivity: A 51-Country survey. American Journal of Preventative Medicine 34 (6): 486-494.

Hallal PC, Andersen LB, Bull FC, Guthold R, Haskell W, Ekelund U 2012 Global physical activity levels: surveillance progress, pitfalls and prospects. Lancet 380: 247-257.

Ites KI, Anderson EJ, Cahill ML, Kearney JA, Post EC, Gilchrist LS 2011 Balance interventions for diabetic peripheral neuropathy: a systematic review. Journal of Geriatric Physical Therapy 34 (3): 109-116

Joubert J, Norman R, Lambert EV, Groenewald P, Schneider M, Bull F, Bradshaw D 2007 Estimating the burden of disease attributable to physical inactivity in South Africa in 2000. South African Medical Journal 97: 725-731.

Mayosi BM, Lawn JE, van Niekerk A, Bradshaw D, Karim SSA, Coovadia HM 2012 Health in South Africa: changes and challenges since 2009. Lancet 380: 2029-2043.

Mensah GA 2008 Ischaemic heart disease in Africa. Heart 94: 836-843.

Lee IM, Shiroma EJ, Lobelo E, Puska P, Blair SN, Katzmarzyk PT 2012 Effects of physical inactivity on major non-communicable diseases worldwide: an analysis of burden of disease and life expectancy. Lancet 380: 219-229.

O'Brien K, Nixon S, Tynann AM, Glazier R 2010 Aerobic exercise intervention for adults living with HIV/AIDS. Cochrane Database of Systematic Reviews, Issue 8. Art No: CD001796. DOI: 10.1002/14651858.CD001796.pub3.

Ramirez-Marrero FA, Rivera-Brown AM, Nazario CM, Rodriquez-Orengo JF, et al. 2008 Self-reported physical activity in Hispanic adults living with HIV: comparison with accelerometer and pedometer. Journal of the association of Nurses in Aids Care 19 (4): 283-294.

Shurie JS \& Deribew A 2010 Assessment of the prevalence of distal symmetrical polyneuropathy and its risk factors among HAART-treated and untreated HIV infected individuals. Ethiopean Medical Journal 48 (2): 85-93.

Statistics South Africa 2010 Statistical Release P0302: Mid-year population estimates 2010. Accessible at www.statssa.gov.za

Steyn K 2008 Hypertension in South Africa. In: Chronic diseases of lifestyle in South Africa since 1995-2005: 1-17. Accessible at http://www.mrc. ac.za/chronic.

Tudor-Locke C, Ainsworth BE, Thompson W, Matthews CE 2002 Comparison of pedometer and accelerometer measures of free-living physical activity. Medicine \& Science in Sports \& Exercise: 2045-2051.

Tudor-Locke C, Basset DR Jr 2004 How many steps/day are enough? Sports Medicine 34 (1): 1-8.

Tudor-Locke C and Lutes L 2009 Why do pedometers work? Sports Medicine 39 (2): 981-993.

UNAIDS 2011 South African Country Overview accessed on 2013-02-11. Accessible at http:// www.unaids.org/en/regionscountries/countries/ southafrica/ 